\title{
Rapamycin induces autophagy to alleviate acute kidney injury following cerebral ischemia and reperfusion via the mTORC1/ATG13/ULK1 signaling pathway
}

\author{
YANG SU $^{1 *}$, JINGXIAO LU ${ }^{1 *}$, PIAN GONG ${ }^{2}$, XIANGUO CHEN $^{3}$, CHAOZHAO LIANG $^{3}$ and JIE ZHANG ${ }^{1,4}$ \\ Departments of ${ }^{1}$ Urology and ${ }^{2}$ Neurosurgery, Renmin Hospital of Wuhan University, Wuhan, Hubei 430060; \\ ${ }^{3}$ Department of Urology, First Affiliated Hospital of Anhui Medical University, Hefei, Anhui 230032; \\ ${ }^{4}$ Department of Urology, Huangshi Central Hospital, Hubei Polytechnic University, Huangshi, Hubei 435000, P.R. China
}

Received March 2, 2018; Accepted September 25, 2018

DOI: $10.3892 / \mathrm{mmr} .2018 .9586$

\begin{abstract}
Acute kidney injury (AKI) is a clinically common and severe complication of ischemia-reperfusion (I/R), associated with high morbidity and mortality rates, and prolonged hospitalization. Rapamycin is a type of macrolide, primarily used for anti-rejection therapy following organ transplantation and the treatment of autoimmune diseases. Rapamycin has been identified to exert a protective effect against AKI induced by renal I/R as an autophagy inducer. However, whether rapamycin preconditioning may relieve AKI following cerebral I/R (CIR) remains to be fully elucidated. The purpose of the present study was to investigate the effects of CIR on the renal system of rats and the role of rapamycin in AKI following CIR. In the present study, a CIR model was established in Sprague-Dawley rats via a 90-min period of middle cerebral artery occlusion and $24 \mathrm{~h}$ reperfusion, and pretreatment with an intraperitoneal injection of rapamycin (dosage: $1 \mathrm{mg} / \mathrm{kg} ; 0.5 \mathrm{~h}$ ) prior to CIR. The levels of serum creatinine and blood urea nitrogen (BUN), and the expression of inflammation-, apoptosis- and autophagy-associated markers were subsequently measured. In addition to certain histopathological alterations to the kidney, it was identified that CIR significantly increased the levels of serum creatinine, BUN, tumor necrosis factor- $\alpha$ and interleukin-1 $\beta$, and significantly induced apoptosis and autophagy. It was observed that rapamycin induced autophagy through the mammalian target of rapamycin complex 1/autophagy-related 13/unc-51 like autophagy activating kinase 1 signaling pathway, and that
\end{abstract}

Correspondence to: Professor Jie Zhang, Department of Urology, Renmin Hospital of Wuhan University, 99 Zhang Zhi-Dong Road, Wuhan, Hubei 430060, P.R. China

E-mail: whuzhangjie@163.com

${ }^{*}$ Contributed equally

Key words: cerebral ischemia-reperfusion, acute kidney injury, autophagy, apoptosis, inflammation rapamycin pre-treatment significantly improved renal function and alleviated renal tissue inflammation and cell apoptosis in rats following CIR. In conclusion, the results suggested that rapamycin may alleviate AKI following CIR via the induction of autophagy.

\section{Introduction}

Acute kidney injury (AKI), characterized by a rapid decrease in renalexcretion function, is ahigh-risk syndrome(1).Its morbidity and mortality rates are progressively increasing worldwide (2), and it is estimated that there are $\sim 13,000,000$ novel cases and $\sim 1700,000$ cases of AKI-associated mortality annually (3). This syndrome has a prevalence of 1-2\% among hospital admissions and occurs in 2-7\% patients during a hospital stay; the mortality rate of patients with AKI in intensive care units may be as high as $50-70 \%$ (4). Furthermore, due to the lack of effective treatments and pharmaceutical interventions, AKI remains a serious challenge to clinicians.

A number of factors may lead to AKI, including infection, sepsis and the use of nephrotoxic drugs (5). In addition, AKI is considered to be a systematic inflammatory response to ischemia-reperfusion (I/R) in various remote organs, including the liver, myocardium, skeletal muscle, intestine and brain (6-10). It was demonstrated that I/R may cause tissue injury by inducing apoptosis. In addition, remote organ I/R-associated AKI is clinically common and intractable; the mechanisms underlying injury induced by organ I/R are multifactorial, including oxidative stress, the generation of free radicals, necrosis, loss of cell polarity, dedifferentiation and the proliferation of viable cells $(11,12)$. Apart from these factors, autophagy is currently an area of interest and has become the focus of an increasing number of studies, providing a novel direction for investigating injury induced by organ I/R.

Autophagy was initially defined as 'self-eating'; in actuality, it is a defense mechanism employed against environmental stress and is critical to a variety of physiological and pathological processes. As the entire process is strictly regulated, it is a conserved process. Autophagy is one type of presentation, which is closely associated with cell survival, although it may additionally be viewed as a type of programmed cell 
death. Among the various cell death pathways, autophagy is considered an inducible and adjustable process that determines cell survival or death (13). Experiments have suggested that I/R-induced injury is a potent trigger of autophagy $(1,14)$, and it has been observed that a high level of autophagy under high atherogenic shear stress may inhibit endothelial cell death and inflammation, thus preventing the development of atherosclerosis. However, whether autophagy has a protective or invasive role in AKI remains controversial. To date, limited previous studies have suggested that the induction of autophagy may cause cell death during AKI; the majority of studies have demonstrated that the activation of autophagy protects against AKI. The appearance of this 'dual role' may depend on the experimental procedures used to investigate organ I/R insults (15).

Therefore, the present study investigated the effects of CIR on the kidneys of rats, and examined the role of rapamycin (an autophagy inducer) in renal injury induced by CIR using a rat model. The aim was to elucidate the mechanism by which AKI develops following CIR, in order to identify novel approaches to its treatment.

\section{Materials and methods}

Animals and environmental conditions. A total of 30 male Sprague-Dawley rats (6 weeks old) weighing 200-250 g were purchased from the Center of Experimental Animals of Wuhan University (Wuhan, China). All animals were randomly assigned to polypropylene cages $(n=5$ per cage) and raised in a specific pathogen-free environment with a natural light-dark cycle (12 $\pm 1 \mathrm{~h}$ light and $12 \pm 1 \mathrm{~h}$ dark). The environmental temperature was maintained at $20-25^{\circ} \mathrm{C}$ and the humidity was maintained at $50-52 \%$. All rats had free access to sterile food and water. The experimental protocol was performed in accordance with the principles and guidelines of the Guide for the Care and Use of Laboratory Animals of the National Institutes of Health (Bethesda, MD, USA). The present study was approved by the Ethics Committee of Renmin Hospital of Wuhan University (Wuhan, China).

Experimental treatment and model construction. All rats were randomly divided into three groups, with 10 rats in each group: Control group (no cerebral ischemia); model group [1.5 h of middle cerebral artery occlusion (MCAO) and $24 \mathrm{~h}$ of reperfusion]; and the pre-treatment group [intraperitoneal injection of $1 \mathrm{mg} / \mathrm{kg}$ rapamycin (Aladdin Biochemical Technology, Shanghai, China) $0.5 \mathrm{~h}$ prior to CIR]. Focal cerebral ischemia was completed under anesthesia with isoflurane (0.5-3\%). During surgery, rectal temperature was maintained at $37 \pm 1^{\circ} \mathrm{C}$ using a heating lamp and a heating pad. The MCAO was performed using a 4.0-monofilament nylon wire (Ethicon, Inc., Cincinnati, $\mathrm{OH}, \mathrm{USA}$ ). Prior to use, the monofilament tip was rounded by heating in a flame. The nylon filaments were held in a suitable location close to the blood vessels for $1.5 \mathrm{~h}$, and subsequently gently retracted to allow reperfusion. In the sham group (control group), the external carotid artery of each rat was exposed, and the incision was sutured immediately without contact with the internal carotid artery. Finally, all rats were sacrificed with excess carbon dioxide, and samples of kidney tissues and blood were collected for further investigation.
Assessment of renal function. Cardiac blood samples $(6 \mathrm{ml}$ for each) were acquired from the rats in each group. The sera were collected by centrifugation at $4^{\circ} \mathrm{C}$ and $2,000 \mathrm{x} \mathrm{g}$ for $15 \mathrm{~min}$ and subsequently stored at $-20^{\circ} \mathrm{C}$ prior to analysis. The serum creatinine and blood urea nitrogen (BUN) levels were measured using a Hitachi 7170s Automatic Biochemical Detector (Hitachi, Ltd., Tokyo, Japan).

Histological examination. The rat kidneys were excised, fixed in $4 \%$ paraformaldehyde for $24 \mathrm{~h}$ at room temperature, embedded in paraffin and cut into $4-\mu \mathrm{m}$ sections for histological staining. The kidney sections were subsequently mounted on glass slides, and hematoxylin-eosin (H\&E) staining was performed for 3-5 min at room temperature for histopathological evaluation. The observed pathological lesions primarily included renal tubular epithelial cell flattening, brush border falling off, cell membrane bleb formation, peritubular/proximal tubule leukocyte infiltration, interstitial edema, cytoplasmic vacuolization, tubular necrosis and tubular lumen obstruction. Pathological scores of $0-5$ points, based on the estimated injury area (\%), were as follows: 0 , normal; 1 , injury area $<10 \%$; 2 , injury area $>10 \%$ but $<25 \%$; 3 , injury area $>25 \%$ but $<50 \%$; 4 , injury area $>50 \%$ but $<75 \%$; 5 , injury area $>75 \%$. For each section, 10 areas were randomly selected to quantitatively assess the extent of AKI using a light microscope (magnification, x200; Olympus Corporation, Tokyo, Japan).

Immunohistochemistry. The paraffin-embedded sections were placed in an oven at $65^{\circ} \mathrm{C}$ for $2 \mathrm{~h}$, dewaxed in xylene and rehydrated. The sections were placed in EDTA buffer for antigen retrieval. Following washing with PBS, the sections were placed in $3 \%$ hydrogen peroxide solution and incubated at room temperature for $10 \mathrm{~min}$, and subsequently blocked with $5 \%$ bovine serum albumin (BSA; Sigma-Aldrich; Merck KGaA, Darmstadt, Germany) for $20 \mathrm{~min}$ at room temperature following PBS-washing and drying. The BSA solution was removed, and $50 \mu$ l diluted primary antibodies against TNF- $\alpha$ (cat. no. ab6671) and IL-1 $\beta$ (cat. no. ab9722; both 1:1,00; Abcam, Cambridge, UK) were added and incubated overnight at $4^{\circ} \mathrm{C}$. Subsequently, 50-100 $\mu$ l biotin-conjugated SP9000 goat-anti-rabbit immunoglobulin G (IgG) secondary antibody (cat. no. TA130016; 1:1,000; OriGene Technologies, Inc., Beijing, China) was added to each section and incubated at $37^{\circ} \mathrm{C}$ for $50 \mathrm{~min}$. Following washing with PBS, 50-100 $\mu$ l DAB solution was added to each section for 5-10 $\mathrm{min}$ at room temperature for the observation of the color under a microscope. Subsequently, the sections were rinsed with distilled water, re-dyed with hematoxylin for $2 \mathrm{~min}$ at room temperature, differentiated with $1 \%$ hydrochloric acid alcohol, and the nucleus was stained blue with ammonia for $10 \mathrm{~min}$ at room temperature. The sections were subsequently placed through a graded ethanol series for 10 min each time, dehydrated and dried, dewaxed with xylene and sealed with neutral gum. Finally, the sections were viewed under a light microscope (magnification, x200; Olympus Corporation).

Terminal deoxynucleotidyl-transferase-mediated dUTP nick end labeling (TUNEL) assay. The TUNEL assay was 
Table I. Serum creatinine and BUN levels of rats from each group.

\begin{tabular}{lccc}
\hline Parameter & CON & CIR & RAPA \\
\hline Scr, $\mu \mathrm{mol} / \mathrm{l}$ & $51.80 \pm 9.88$ & $70.69 \pm 11.94^{\mathrm{a}}$ & $58.07 \pm 10.08^{\mathrm{b}}$ \\
BUN, $\mathrm{mmol} / 1$ & $2.85 \pm 0.56$ & $4.03 \pm 0.78^{\mathrm{a}}$ & $3.09 \pm 0.61^{\mathrm{b}}$ \\
\hline
\end{tabular}

Serum creatinine and BUN were measured following $90 \mathrm{~min}$ of cerebral ischemia and $24 \mathrm{~h}$ of reperfusion for rats in each group. The results are presented as the mean \pm standard deviation. $n=10$ /group. ${ }^{\mathrm{a}} \mathrm{P}<0.05$ CIR vs. CON; ${ }^{\mathrm{b}} \mathrm{P}<0.05 \mathrm{RAPA}$ vs. CIR. CON, control; CIR, cerebral ischemia-reperfusion; RAPA, CIR + rapamycin pre-treatment; Scr, serum creatinine; BUN, blood urea nitrogen.

performed according to the manufacturer's protocol (Roche Diagnostics, Indianapolis, IN, USA). The renal tissue sections were placed in proteinase $\mathrm{K}$ solution $(20 \mu \mathrm{g} / \mathrm{ml})$, hydrolyzed for $15 \mathrm{~min}$ at room temperature to remove the tissue protein, and subsequently placed into $10 \mathrm{mM}$ sodium citrate buffer (pH 6.0) for $10 \mathrm{~min}$ for antigen retrieval. The sections were incubated with $3 \%$ hydrogen peroxide for 30 min to block endogenous peroxidase activity. Following washing with PBS, the sections were incubated with $1.5 \%$ normal goat serum (Beijing Solarbio Science \& Technology, Ltd., Beijing, China) for $30 \mathrm{~min}$ at room temperature, followed by incubation with TUNEL reaction mixture overnight at $4^{\circ} \mathrm{C}$. Following washing with PBS, the sections were incubated with $0.05 \%$ DAB for $5 \mathrm{~min}$ at room temperature. Finally, the sections were mounted with neutral balsam, and the results were examined under a light microscope (magnification, x200; Olympus Corporation). The cells with positive TUNEL staining (brown staining in the nucleus) were counted in 10 different fields per section. The results are expressed as the mean number of TUNEL-positive cells in each group.

Western blot analysis. The renal tissue proteins were extracted from the samples of each group. The tissues were homogenized in a lysis buffer provided by Shanghai Biyuntian Bio-Technology Co., Ltd. (Shanghai, China) with a polytron homogenizer (IKA GmbH, Königswinter, Germany) on ice. The lysates were subsequently collected, and the concentrations of protein were detected with a bicinchoninic acid protein assay. Equal quantities of total protein $(40 \mu \mathrm{g})$ were loaded into each well, resolved via $15 \%$ SDS-PAGE, fractionated by electrophoresis and transferred onto polyvinylidene difluoride membranes. The membranes were blocked with $5 \%$ non-fat milk dissolved in Tris-buffered saline with Tween 20 for $1 \mathrm{~h}$ at room temperature. The following primary antibodies were incubated with the membranes overnight at $4^{\circ} \mathrm{C}$ : Anti-microtubule-associated protein 1 light chain $3 \beta$ (LC3B; cat. no. ab192890), anti-Beclin-1 (cat. no. ab207612), anti-p62 (cat. no. ab155686), anti-caspase-9 (cat. no. ab2013), anti-caspase-3 (cat. no. ab184787), anti-cleaved caspase-3 (cat. no. ab184787), anti-B-cell lymphoma (Bcl)-2 (cat. no. ab196495; all 1:2,000; Abcam), anti-autophagy-related 13 (Atg13; cat. no. 13273), anti-mammalian target of rapamycin complex 1 (mTORC1; cat. no. 2587), anti-unc-51 like autophagy activating kinase 1 (ULK1; cat. no. 8054) and anti-phosphorylated (p-)ULK1 (cat. no. 14202; all 1:1,000; Cell Signaling Technology, Inc., Danvers, MA, USA). Subsequently, a goat-anti-rabbit fluorescently-labeled secondary antibody (cat. no. C51007; 1:15,000; LI-COR Biosciences, Lincoln, NE, USA) conjugated to horseradish peroxidase was used for $1 \mathrm{~h}$ at room temperature to identify the primary antibodies. The protein bands were detected with a two-color infrared imaging system (Odyssey; LI-COR Biosciences, Lincoln, NE, USA). The relative band intensity was quantified using Quantity One 4.6.2 software (Bio-Rad Laboratories, Inc., Hercules, CA, USA). GAPDH was used as an internal reference.

RNA isolation and reverse transcription-quantitative polymerase chain reaction $(R T-q P C R)$ analysis. Total RNA was isolated from the kidney tissues using TRIzol ${ }^{\circledR}$ (Invitrogen; Thermo Fisher Scientific, Inc., Waltham, MA, USA), following the manufacturer's protocol. RT $\left(37^{\circ} \mathrm{C}\right.$ for $15 \mathrm{~min}$ and $85^{\circ} \mathrm{C}$ for $5 \mathrm{sec}$ ) was performed using a PrimeScript RT Reagent kit (Takara Bio, Inc., Otsu, Japan). Amplification was performed with a 7500 Real-Time PCR system (Applied Biosystems; Thermo Fisher Scientific, Inc.). The reaction mixture (total $20 \mu \mathrm{l}$ ) contained $2 \mu \mathrm{l}$ cDNA, $10 \mu \mathrm{M}$ primers and $10 \mu \mathrm{l}$ 2X SYBR Premix Ex Taq II (Takara Bio, Inc.). The primer sequences were designed using the Primer Express 2.0 software package (Applied Biosystems; Thermo Fisher Scientific, Inc.), and were as follows: TNF- $\alpha$ forward, 5'-CTTCTCATTCCTGCTCGT GG-3' and reverse, 5'-CGGGCTTGTCACTCGAGTTT-3'; IL-1 $\beta$ forward, 5'-GGCAGTGTCACTCATTGTGG-3' and reverse, 5'-CTAGCAGGTCGTCATCATCCC-3'; GAPDH forward, 5'-CGCTAACATCAA ATGGGGTG-3' and reverse, 5'-TTGCTGACAATCTTGAGGGAG-3'.

The thermocycling conditions were as follows: $95^{\circ} \mathrm{C}$ for $30 \mathrm{sec}, 40$ cycles of denaturation at $95^{\circ} \mathrm{C}$ for $5 \mathrm{sec}$ and extension at $60^{\circ} \mathrm{C}$ for $40 \mathrm{sec}$. All samples were run in triplicate, and the melting curves of all products were analyzed. Quantitative measurements were determined using the $2^{-\Delta \Delta \mathrm{Cq}}$ method (16). GAPDH was used as the internal control.

Immunofluorescence. The paraffin-embedded sections were fixed in $100 \%$ acetone for $20 \mathrm{~min}$ at room temperature. Following washing with PBS and antigen retrieval, the sections were placed in a $3 \% \mathrm{H}_{2} \mathrm{O}_{2}$-methanol solution and incubated at room temperature for $10 \mathrm{~min}$. The sections were subsequently blocked with 5\% BSA (Sigma-Aldrich; Merck $\mathrm{KGaA}$, Darmstadt, Germany) for $20 \mathrm{~min}$ at room temperature following PBS-washing and drying. The renal tissue sections were incubated with a monoclonal primary antibody against LC3B (cat. no. ab48394; 1:1,00; Abcam) at $4^{\circ} \mathrm{C}$ overnight following PBS washing. The sections were subsequently incubated with a fluorescein isothiocyanate-labeled goat-anti-rabbit $\operatorname{IgG}$ (cat. no. ab6717; 1:2,000; Abcam) at $37^{\circ} \mathrm{C}$ for $1 \mathrm{~h}$ in the dark. Finally, the sections were sealed with glycerol and observed using a fluorescence microscope (magnification, x200; Olympus Corporation). The quantification was analyzed using the Image Pro-Plus 6.0 system (Media Cybernetics, Inc., Rockville, MD, USA).

Statistical analysis. All experiments were repeated three times independently. Data are presented as the mean \pm standard 
A

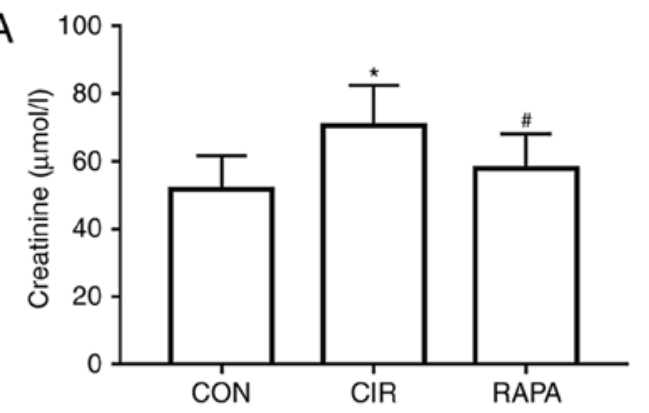

B

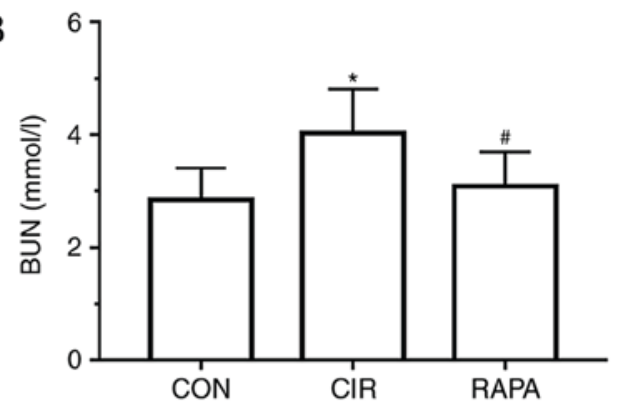

C

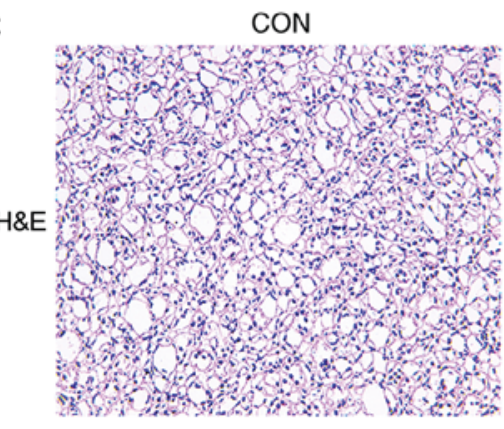

CIR

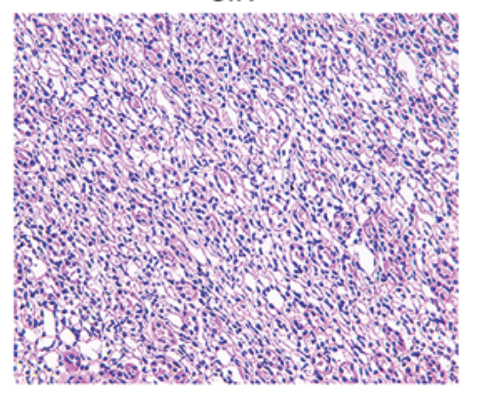

RAPA
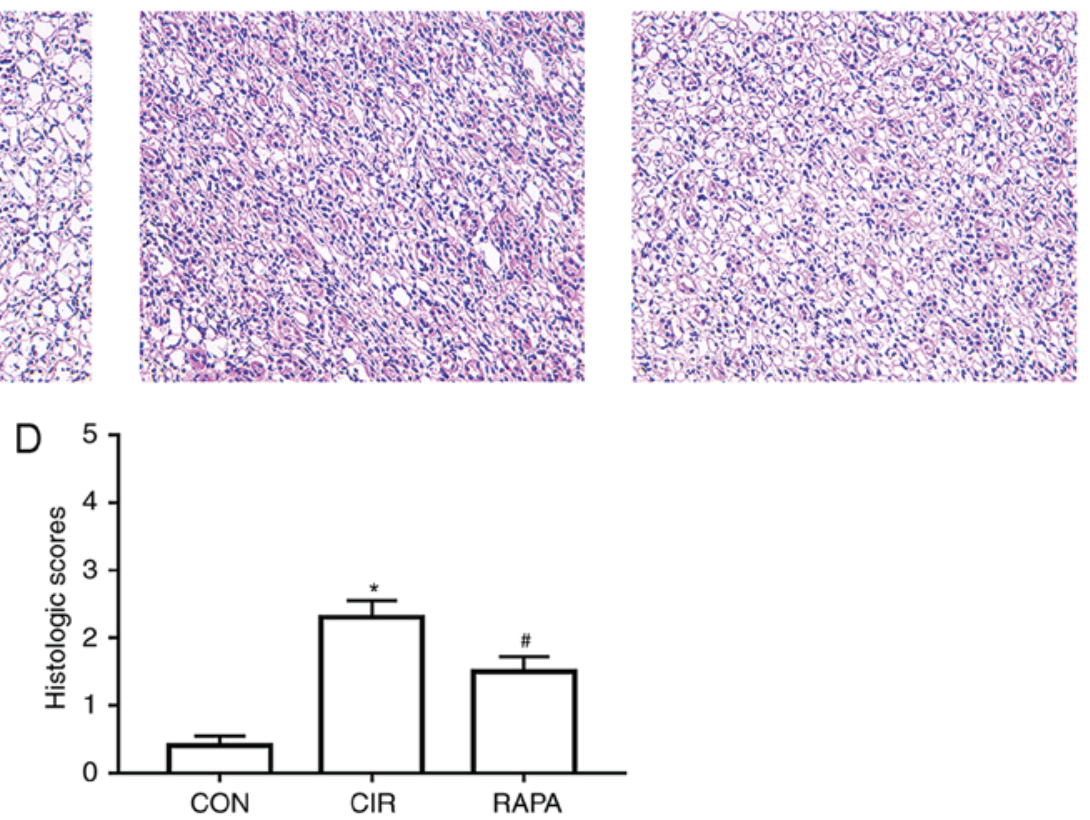

Figure 1. Changes in renal function and histopathology in rats from each group. Levels of (A) serum creatinine and (B) BUN were measured via blood biochemistry. (C) Representative images of pathological alterations in the kidney tissues of rats (H\&E staining; x200 magnification). (D) Degrees of renal tubulointerstitial injury were evaluated using pathological scoring criteria. The results are presented as the mean \pm standard deviation. $\mathrm{n}=10 / \mathrm{group}$. ${ }^{*} \mathrm{P}<0.05$ CIR vs. CON; " $\mathrm{P}<0.05$ RAPA vs. CIR. CON, control group; CIR, cerebral ischemia-reperfusion; RAPA, rapamycin pre-treatment prior to CIR; BUN, blood urea nitrogen; H\&E, hematoxylin and eosin.

deviation. GraphPad Prism v5.0 (GraphPad Software, Inc., La Jolla, CA USA) was used to analyze the results using one-way analysis of variance and Student's t-test. Multiple comparisons between the groups were performed using Tukey's method as the post hoc test. $\mathrm{P}<0.05$ was considered to indicate a statistically significant difference.

\section{Results}

CIR causes AKI in rats. To assess renal alterations following CIR in the present study, histopathological alterations were assessed in the kidneys of the rats, and indices reflective of renal function were measured, including serum creatinine and BUN, following $90 \mathrm{~min}$ of MCAO and $24 \mathrm{~h}$ of reperfusion. The results demonstrated that the levels of serum creatinine and BUN were significantly increased following CIR $(\mathrm{P}<0.05$; Table I; Fig. 1A and B). In addition, compared with the control group, falling off the brush border of renal tubular epithelial cells, renal tubular dilation, tube type in lumen, renal tubular necrosis and increased inflammatory cell infiltration around the tubules were observed in the CIR group, as observed in the H\&E-stained sections (Fig. 1C). The histological score was additionally significantly increased in the CIR group compared with the control group ( $\mathrm{P}<0.05$; Fig. 1D).

Rapamycin ameliorates CIR-induced renal dysfunction in rats. To determine the role of rapamycin in CIR-induced AKI in rats, rapamycin was administered via intraperitoneal injection to the rats prior to CIR. It was identified that, compared with the CIR group, the levels of serum creatinine and BUN in the rapamycin pre-treatment group were significantly reduced $(\mathrm{P}<0.05$; Fig. 1A and $\mathrm{B})$. It was additionally demonstrated that the kidney histopathological alterations were markedly improved in the rapamycin pre-treatment group compared with the CIR group (Fig. 1C); the histological score was additionally significantly decreased $(\mathrm{P}<0.05$; Fig. 1D).

Rapamycin alleviates CIR-induced renal inflammation in rats. Renal injury is closely associated with the expression of certain inflammatory mediators. In order to determine the effects of CIR on renal inflammation in rats and assess whether rapamycin may regulate this inflammation, the present study examined how the expression of TNF- $\alpha$ and IL-1 $\beta$ altered with rapamycin pre-treatment. The results of 

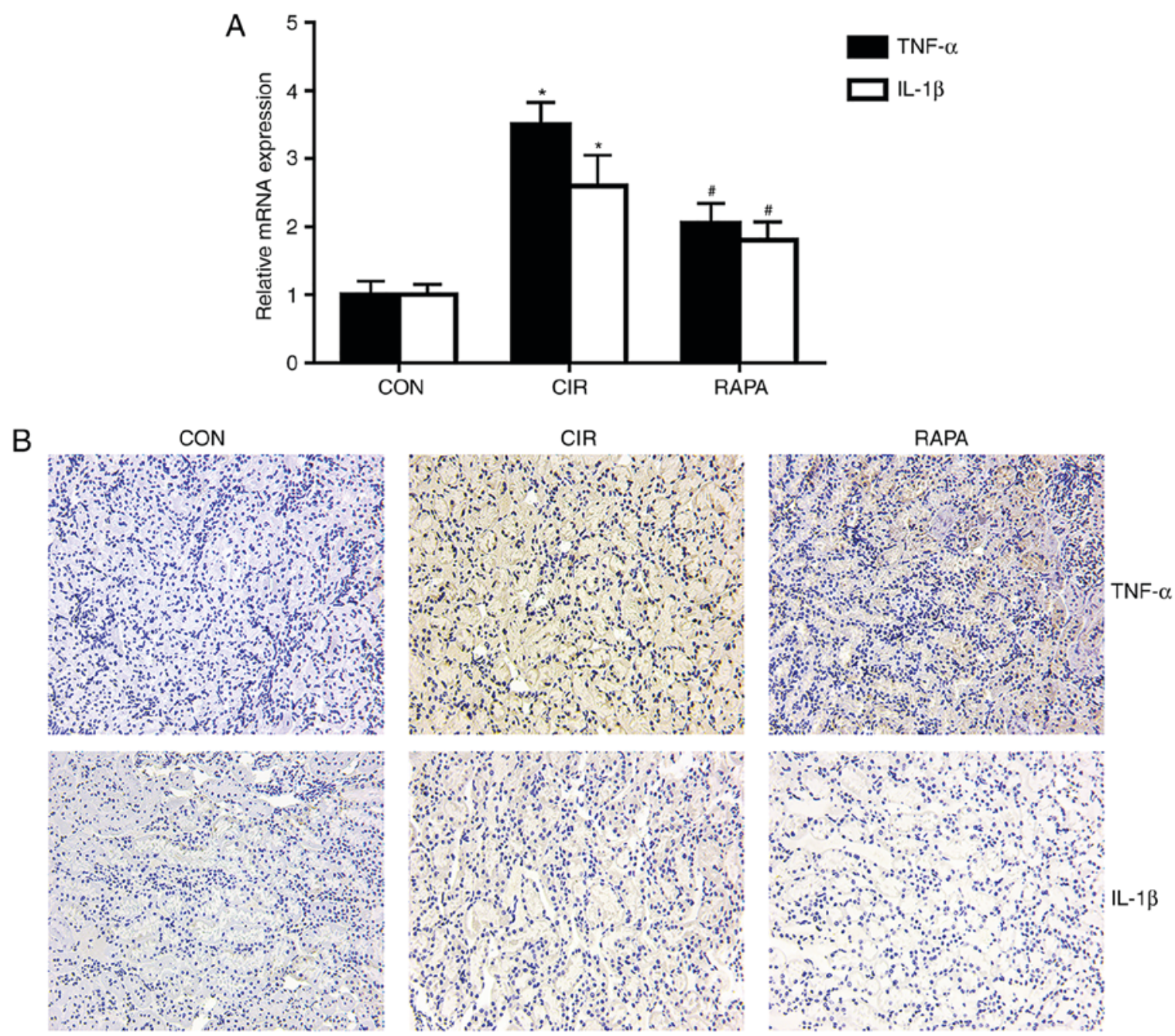

Figure 2. Renal inflammation in rats from each group. (A) Relative mRNA expression levels of TNF- $\alpha$ and IL-1 $\beta$ in the kidney tissues of rats were evaluated by reverse transcription-quantitative polymerase chain reaction analysis. (B) Representative images of the expression of TNF- $\alpha$ and IL- $1 \beta$ from immunohistochemistry (x200 magnification). The results are presented as the mean \pm standard deviation. $\mathrm{n}=10 / \mathrm{group}$. ${ }^{*} \mathrm{P}<0.05 \mathrm{CIR}$ vs. CON; ${ }^{*} \mathrm{P}<0.05 \mathrm{RAPA}$ vs. CIR CON, control group; CIR, cerebral ischemia-reperfusion; RAPA, rapamycin pre-treatment prior to CIR; TNF- $\alpha$, tumor necrosis factor- $\alpha$; IL-1 $\beta$, interleukin-1 $\beta$.

the RT-qPCR analysis $(\mathrm{P}<0.05$; Fig. 2A) and immunohistochemistry (Fig. 2B) demonstrated that the expression levels of TNF- $\alpha$ and IL- $1 \beta$ in the kidney tissues were significantly increased in the CIR group compared with the control group, whereas rapamycin pre-treatment significantly reversed these effects $(\mathrm{P}<0.05$; Fig. 2$)$.

Rapamycin suppresses CIR-induced renal apoptosis in rats. Apoptosis is a pathological process involved in the development of AKI. To examine the effects of CIR on renal apoptosis in rats and the role of rapamycin in AKI following CIR, the present study assessed the protein expression of Bcl-2 and cleaved caspase- 3 , which are commonly used markers of apoptosis, and the expression of caspase- 9 and caspase- 3 . The western blot analysis demonstrated that, compared with the control group, the expression of cleaved caspase-3 was significantly increased and the expression of Bcl-2 was significantly decreased in the CIR group $(\mathrm{P}<0.05$; Fig. 3A-E). However, a significant increase in the expression of $\mathrm{Bcl}-2$ and significant decrease in the expression of cleaved caspase- 3 was observed in the rapamycin pre-treatment group, compared with the CIR group $(\mathrm{P}<0.05$; Fig. 3A-E). In addition, the
TUNEL staining results demonstrated that the number of TUNEL-positive cells in the CIR group was higher compared than that in the control group (Fig. 3F), which was corroborated by quantitative analysis $(\mathrm{P}<0.05$; Fig. $3 \mathrm{G})$. However, rapamycin pre-treatment significantly decreased the number of TUNEL-positive cells, compared with the number in the CIR group ( $\mathrm{P}<0.05$; Fig. $3 \mathrm{~F}$ and $\mathrm{G})$.

Rapamycin enhances CIR-induced renal autophagy in rats. To elucidate the effects of CIR on renal autophagy in rats, and further assess the role of rapamycin in CIR-induced AKI, rapamycin was administered via intraperitoneal injection to the rats prior to CIR and the expression of numerous critical autophagy markers, including LC3, Beclin-1 and p62, were measured. As presented in Fig. 4A-D, the expression levels of LC3B, a more common form of LC3, and Beclin-1 were significantly increased and the expression of p62 was significantly decreased in the CIR group, compared with the control group, as determined by western blot analysis $(\mathrm{P}<0.05)$. A similar trend was observed for the expression of LC3B in the kidney tissues of rats via fluorescence microscopy and quantitative analysis ( $\mathrm{P}<0.05$; Fig. $4 \mathrm{E}$ and $\mathrm{F})$. Additionally, 
A

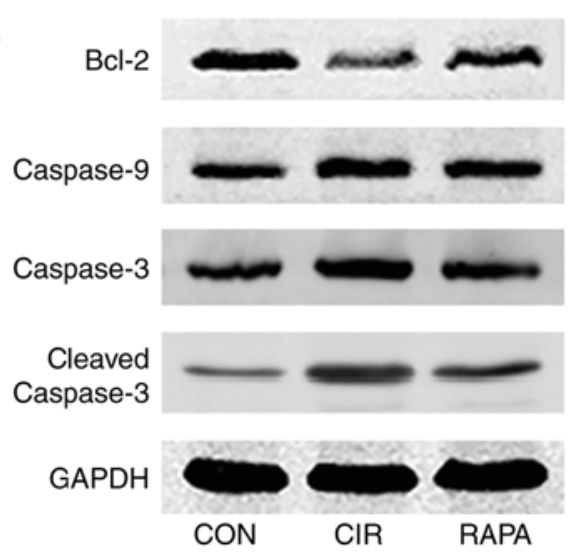

$\mathrm{F}$

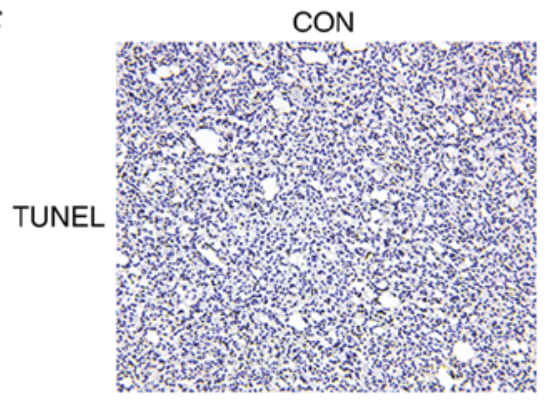

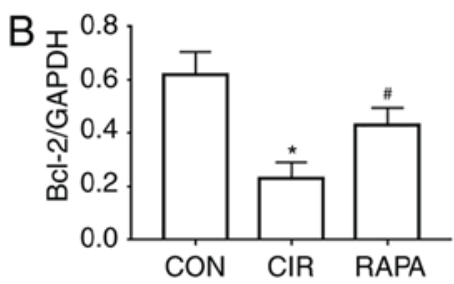
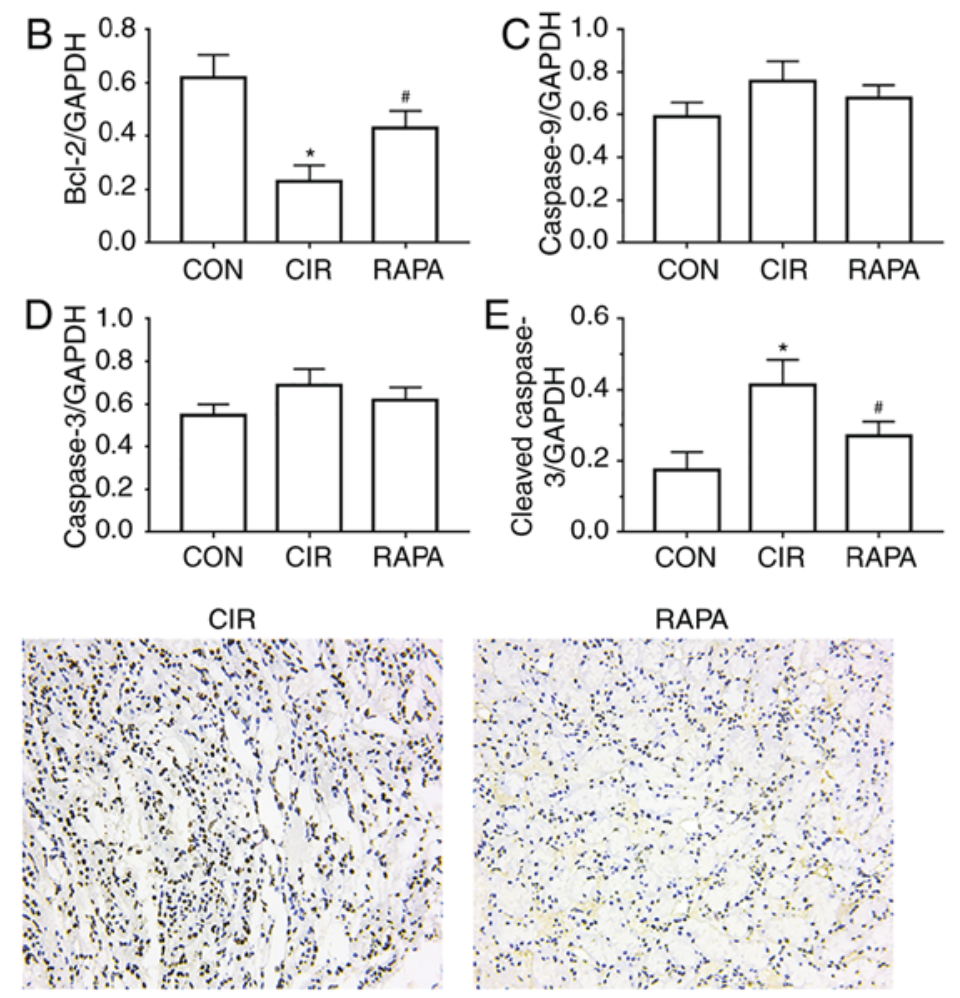

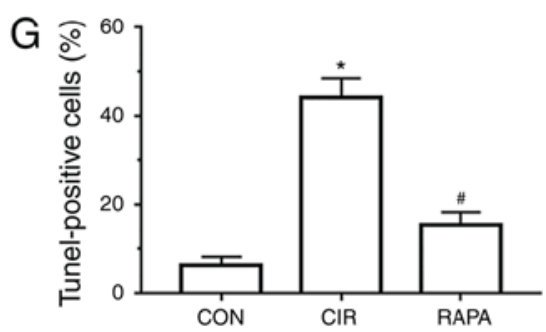

Figure 3. Renal cell apoptosis in rats from each group. (A) Representative images of protein expression of Bcl-2, caspase-9, caspase-3 and cleaved caspase-3, obtained by western blot analysis. Relative protein expression levels of (B) Bcl-2, (C) caspase-9, (D) caspase-3 and (E) cleaved caspase-3 were determined by quantitative analysis. (F) Representative images of cell apoptosis in the kidney tissues of rats (TUNEL staining; x200 magnification). (G) Percentage of apoptotic cells in each group (quantitative analysis). The results are presented as the mean \pm standard deviation. $n=10 /$ group. ${ }^{\mathrm{P}}<0.05 \mathrm{CIR}$ vs. CON; ${ }^{*} \mathrm{P}<0.05$ RAPA vs. CIR. CON, control group; CIR, cerebral ischemia-reperfusion; RAPA, rapamycin pre-treatment prior to CIR; Bcl-2, B-cell lymphoma 2.

the expression levels of LC3B and Beclin-1 were increased further and the expression of p62 was decreased further in the rapamycin pre-treatment group compared with the CIR group, as determined by western blot analysis $(\mathrm{P}<0.05$; Fig. 4A-D). Similarly, compared with the CIR group, fluorescence microscopy and quantitative analysis demonstrated that LC3B levels were further significantly increased in the rapamycin pre-treatment group $(\mathrm{P}<0.05$; Fig. $4 \mathrm{E}$ and $\mathrm{F})$.

Rapamycin regulates renal autophagy in rats via the mTORC1/ULK1/Atg13 signaling pathway. To further examine the potential mechanism involved in rapamycin relieving AKI following CIR via the activation of autophagy, the levels of a number of crucial autophagy-associated proteins, including mTORC1, Atg13, ULK1 and p-ULK1, were assessed. The results of the western blot and quantitative analyses suggested that the expression levels of Atg13 and ULK1 were significantly increased, and the expression levels of mTORC1 and p-ULK1 were significantly decreased in the rapamycin pre-treatment group, compared with expression levels in the CIR group (P<0.05; Fig. 5).

\section{Discussion}

It is well known that the injury of one organ may cause alterations in another distal organ. The interaction between the liver and kidney is known as hepato-renal syndrome, and that between the heart and kidney is known as cardio-renal syndrome. Pulmonary-renal, intestinal-kidney and oculo-cerebro-renal syndromes have additionally been described (17-21). Therefore, as a systematic response, CIR is considered not only to cause brain tissue damage; however, additionally to induce damage to distant organs, including the kidney. Tsagalis et al (22) identified that AKI was a common complication following acute stroke, including ischemic stroke, and demonstrated that AKI was an independent predictor of early and long-term mortality following acute stroke. Khatri et al (23) observed that renal dysfunction was induced by acute ischemic stroke, and that it was associated with a longer hospital stay and increased mortality rate. In the present study, numerous histological alterations were identified in rat kidneys following CIR, including widespread renal tubular necrosis, inflammatory cell infiltration and tubular 
A
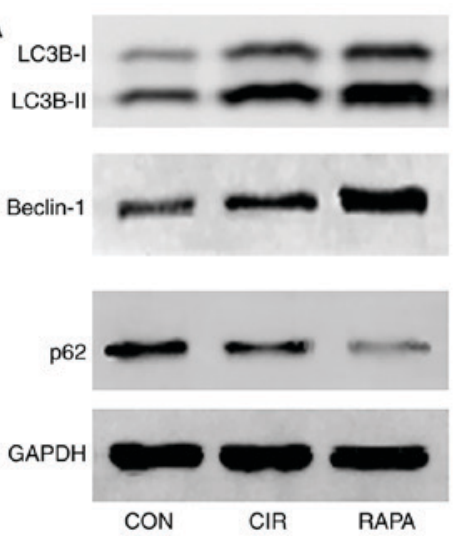

E
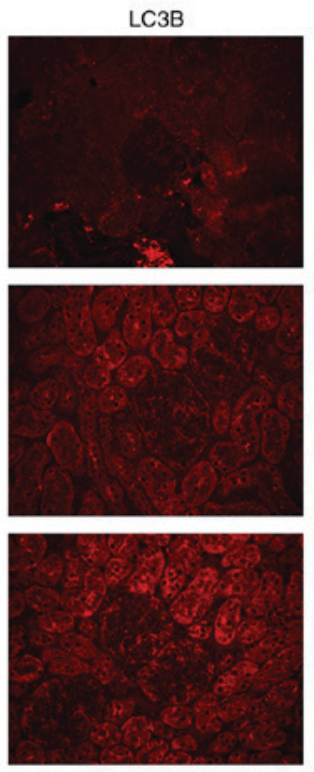
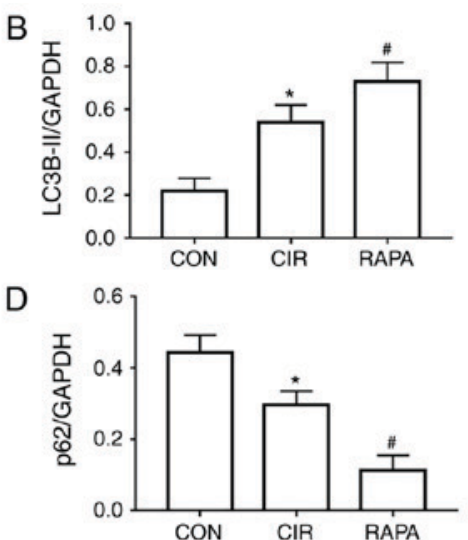

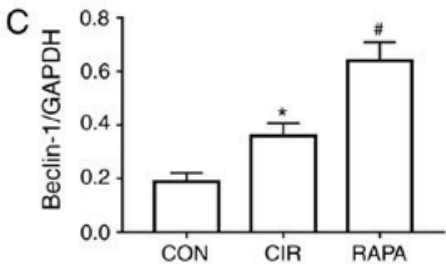

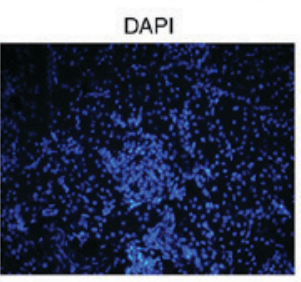
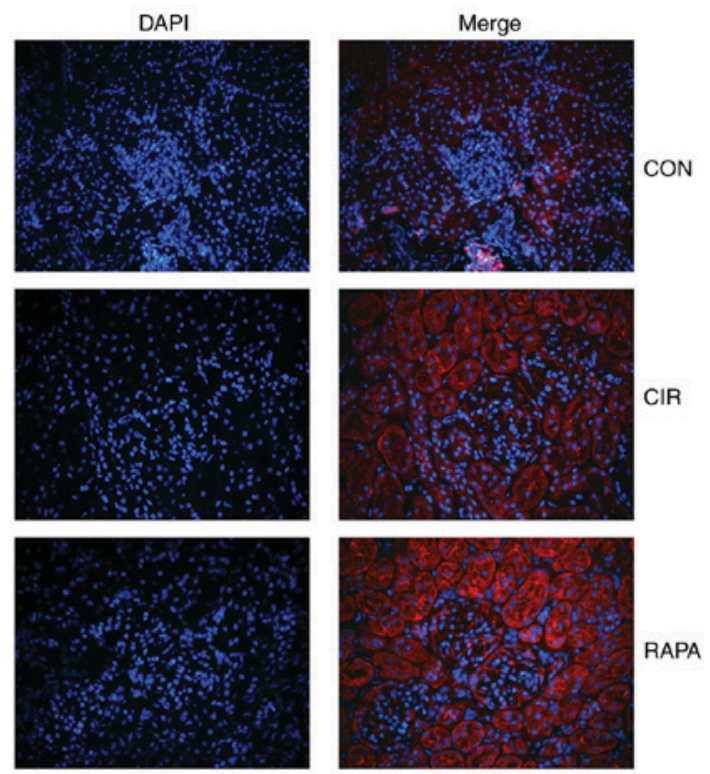

$\mathrm{F}$

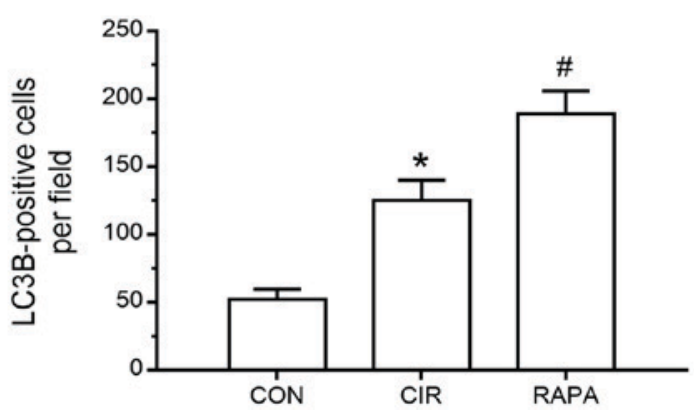

Figure 4. Renal cell autophagy in rats from each group. (A) Representative images of the protein expression of LC3B, Beclin-1 and p62, as determined by western blot analysis. Relative protein expression of (B) LC3B-II, (C) Beclin-1 and (D) p62, as determined through quantitative analysis. (E) Representative fluorescence microscopy images of LC3B (magnification, $\mathrm{x} 200$ ). (F) Number of LC3B-positive cells in each group (quantitative analysis). The results are presented as the mean \pm standard deviation. $\mathrm{n}=10$ /group. ${ }^{*} \mathrm{P}<0.05 \mathrm{CIR}$, vs. CON; ${ }^{"} \mathrm{P}<0.05 \mathrm{RAPA}$, vs. CIR. CON, control group; CIR, cerebral ischemia-reperfusion; RAPA, rapamycin pre-treatment prior to CIR; LC3B, microtubule-associated protein 1 light chain $3 \beta$.

dilatation, among others. Furthermore, rats in the CIR group exhibited renal dysfunction, which was reflected in the significant elevation of serum creatinine and BUN levels, compared with the control group.

AKI is a persistent clinical problem associated with high mortality rates and healthcare costs. The incidence of AKI has been increasing, and is likely to increase even further in the future due to the aging population and the emergence of comorbidities (22). AKI may cause an inflammatory response and apoptosis within the kidney (24). Inflammation is a primary factor involved in the progression of AKI; the acute inflammatory response is characterized by the activation of inflammatory cells and the excessive secretion of pro-inflammatory cytokines, including TNF- $\alpha$ and IL-1 $\beta$ (25). Nongnuch et al (26) demonstrated that acute cerebral injury may cause AKI and trigger an inflammatory cascade in the kidney. In the present study, compared with the control group, increased inflammatory cell infiltration was identified in the kidney sections from the CIR group, as determined by H\&E staining, and increased secretion of TNF- $\alpha$ and IL-1 $\beta$ was 
A
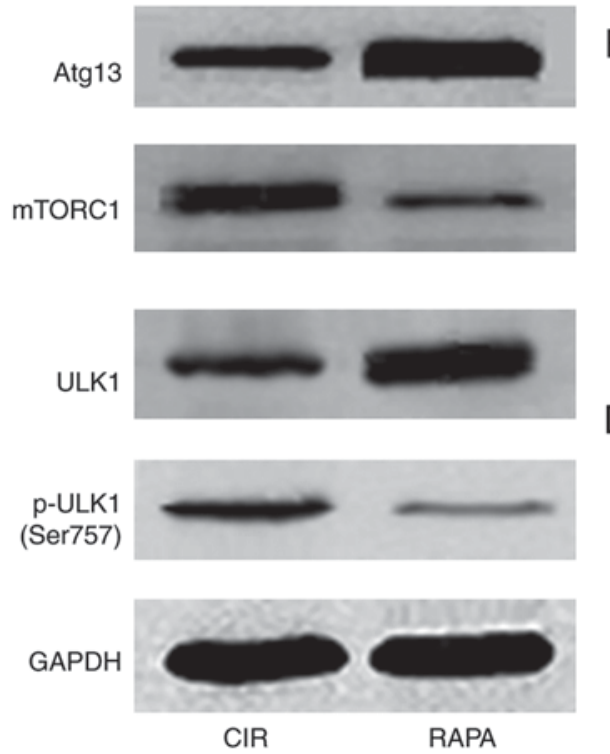

$B$

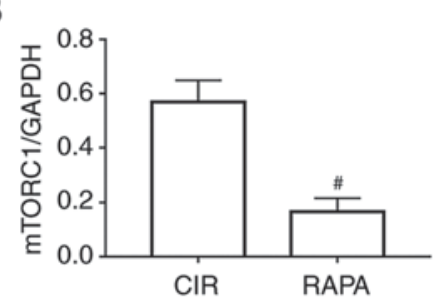

D

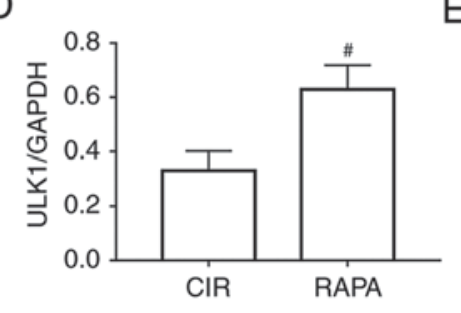

C

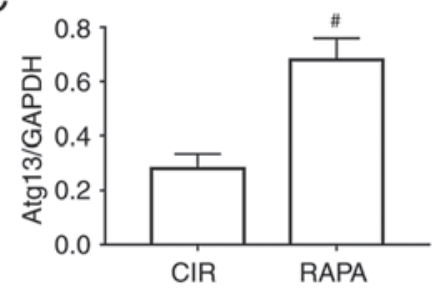

E

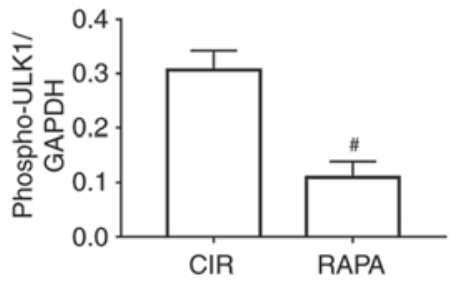

Figure 5. Involvement of a relevant signaling pathway in rapamycin relieving CIR-induced acute kidney injury through the activation of autophagy. (A) Representative images of the protein expression of mTORC1, Atg13, ULK1 and p-ULK1, as determined by western blot analysis. Relative expression of (B) mTORC1, (C) Atg13, (D) ULK1 and (E) p-ULK1, as determined via quantitative analysis. The results are presented as the mean \pm standard deviation. $\mathrm{n}=10$ /group. ${ }^{\text {P }}<0.05$ RAPA vs. CIR. CIR, cerebral ischemia-reperfusion; RAPA, rapamycin pre-treatment prior to cerebral ischemia-reperfusion; mTORC1, mammalian target of rapamycin complex; Atg-13, autophagy-related 13; ULK1, unc-51 like autophagy activating kinase 1; p-ULK1, phosphorylated-ULK1.

observed in the CIR group, as demonstrated by immunohistochemistry. Apoptosis is another central mechanism in AKI; it is an organized process regulating the development and homeostasis of multiple organisms, and is a type of autonomic and programmed cell death pathway regulated by genes (27). Apoptosis is critical in various physiological processes and pathological conditions, and involves the expression of apoptosis-associated genes, including Bcl-2 and caspase-3 (28). These proteins either promote or inhibit apoptosis, and the imbalance between pro- and anti-apoptotic genes may be a decisive factor. Bcl-2 family proteins are potent regulators of apoptosis; it is increasingly believed that Bcl-2 may inhibit cell death from a wide variety of pathogenic stimuli. It may additionally inhibit mitochondrial membrane potential and decrease caspase- 3 activation, in addition to inhibiting apoptosis via its binding to pro-apoptotic proteins (28). Bcl-2 is a substrate of caspase-3, and may thus be hydrolyzed by caspase-3. Regarding the activation of proteases, a proteolytic cascade of effector caspases is directly responsible for the execution phase of apoptosis (28). The 'executioner' caspase-3 is activated by the 'initiator' caspase-9, resulting in cell death; therefore, caspase-3 may promote apoptosis $(29,30)$. In the present study, it was identified that CIR increased the protein expression of cleaved caspase-3 (an activated form of caspase-3) and inhibited the protein expression of Bcl-2 in rat kidney tissues, compared with the control group, as determined by western blot analysis. Furthermore, the numbers of TUNEL-positive cells were significantly increased in the CIR group.

The basic pathogenesis of AKI is multifactorial, including ischemia, hypoxia, nutrient and growth factor deprivation, energy depletion, oxidant injury, endoplasmic reticulum stress and other factors; these stimuli may drive autophagy (31). Among those that are activated as part of the renal stress response to organ $\mathrm{I} / \mathrm{R}$, autophagy has become the focus of numerous investigations (31). Autophagy is an evolutionarily conserved multistep process that involves the degradation of intracellular organelles, proteins and other macromolecules by lysosomal hydrolytic enzymes (32). The degraded cellular contents are utilized for the synthesis of novel macromolecules and organelles. Under normal physiological conditions, a basal level of autophagy maintains cellular homeostasis (32). Under pathological conditions, external stressors contribute to the induction of autophagy (33). In cell and animal models of I/R-induced injury, it has been demonstrated that autophagy is activated (34). As no available effective therapies for AKI are available, one of the increasingly recognized and potential therapeutic targets is cellular autophagy. A number of previous studies demonstrated the role of autophagy in I/R-induced AKI. Chien et al (35) identified that autophagy may ameliorate AKI caused by I/R. Hsiao et al (36) suggested that autophagy is beneficial in AKI due to sepsis by cecal ligation and puncture, and that the decline of autophagy contributed to proximal tubular dysfunction in late-stage sepsis. Kimura et al (37) used proximal tubule-specific Atg5-knockout mice to demonstrate that autophagy was reno-protective following I/R. Additionally, Sun et al (38) observed that octreotide may reduce AKI following hepatic $\mathrm{I} / \mathrm{R}$ in a rat model via the induction of autophagy. Zhang et al (39) demonstrated that niclosamide may attenuate inflammation by inducing autophagy in a rat model of renal I/R.

As the consequences of autophagy in CIR-induced AKI have not been investigated, to the best of our knowledge, and based on the aforementioned studies, it was hypothesized that autophagy may additionally be important in CIR-induced AKI. However, whether it provides protection or aggravates AKI following CIR remains to be elucidated. A frequently used autophagy inducer is rapamycin, a macrolide antibiotic originally used for antifungal therapy. However, it may additionally be used to regulate autophagy and maintain cell 
metabolism. Zhang et al (14) demonstrated that rapamycin may be a promising therapy for I/R and AKI. Cui et al (40) suggested that rapamycin may ameliorate gentamicin-induced AKI by enhancing autophagy in miniature pig models. Luo et al (41) identified that rapamycin inhibited vascular smooth muscle cell senescence via inducing autophagy. In the present study, it was identified that rapamycin pre-treatment prior to CIR attenuated renal pathological alterations and improved renal function, and the number of inflammatory cells around the renal tubules was significantly reduced in the rapamycin pre-treatment group compared with the CIR group. Furthermore, rapamycin pre-treatment suppressed the expression of TNF- $\alpha$ and IL-1 $\beta$ in rat kidney tissues. Compared with the CIR group, rapamycin pre-treatment increased the protein expression of $\mathrm{Bcl}-2$ and decreased the protein expression of cleaved caspase-3; additionally, the rapamycin pre-treatment group exhibited fewer TUNEL-positive cells compared with the CIR group. It was additionally observed that the protein expression levels of LC3B and Beclin-1 were significantly increased, whereas the protein expression of p62 was significantly inhibited in the CIR group, compared with the control group; this effect of autophagy was considered to be a limited self-protection response to stress. Rapamycin pre-treatment followed by CIR resulted in the increased induction of LC3B and Beclin-1 proteins and inhibition of p62 protein, mediated via the mTORC1/ATG13/ULK1 signaling pathway. Fluorescence microscopy analysis additionally demonstrated that the expression of LC3B in rat kidney tissues was induced by CIR and further enhanced by rapamycin. Therefore, the results demonstrated that CIR may cause AKI, and that rapamycin pre-treatment may improve renal function, reduce renal inflammation and apoptosis, and further activate CIR-induced autophagy in the kidneys of rats via the mTORC1/ATG13/ULK1 signaling pathway.

In conclusion, rapamycin may relieve CIR-induced AKI by activating autophagy through the mTORC1/ATG13/ULK1 signaling pathway. These results are likely to assist in further elucidating the pathogenesis of AKI following CIR and may provide a promising treatment approach for this condition. However, the specific underlying mechanisms require further investigation.

\section{Acknowledgements}

The authors would like to thank Professor Zhihua Wang of the Central Laboratory of Renmin Hospital of Wuhan University for providing relevant experimental facilities and technical support.

\section{Funding}

The present study was supported by the National Natural Science Foundation of China (grant nos. 81470923, 81770078 and 81770688).

\section{Availability of data and materials}

The datasets used and/or analyzed during the current study are available from the corresponding author on reasonable request.

\section{Authors' contributions}

YS, XC, CL and JZ designed the study. YS, JL and PG performed the experiments. JL and XC gathered the experimental data. XC and YS analyzed the experimental data. YS drafted the manuscript. XC, CL and JZ revised the paper for intellectual content. All authors read and approved the final manuscript.

\section{Ethics approval and consent to participate}

The experimental protocol was performed in accordance with the principles and guidelines of the Guide for the Care and Use of Laboratory Animals of the National Institutes of Health. The present study was approved by the Ethics Committee of Renmin Hospital of Wuhan University.

\section{Patient consent for publication}

Not applicable.

\section{Competing interests}

The authors declare that they have no competing interests.

\section{References}

1. Melk A, Baisantry A and Schmitt R: The yin and yang of autophagy in acute kidney injury. Autophagy 12: 596-597, 2016.

2. Ronco C and Chawla LS: Acute kidney injury: Kidney attack must be prevented. Nat Rev Nephrol 9: 198-199, 2013.

3. Mehta RL, Cerdá J, Burdmann EA, Tonelli M, Garcia-Garcia G, Jha V, Susantitaphong P, Rocco M, Vanholder R, Sever MS, et al: International Society of Nephrology's 0by 25 initiative for acute kidney injury (zero preventable deaths by 2025): A human rights case for nephrology. Lancet 385: 2616-2643, 2015.

4. Bellomo R, Kellum JA and Ronco C: Acute kidney injury. Lancet 380: 756-766, 2012.

5. Dirkes S: Sepsis and inflammation: Impact on acute kidney injury. Nephrol Nurs J 40: 125-132, 2013.

6. Lee HT, Park SW, Kim M and D'Agati VD: Acute kidney injury after hepatic ischemia and reperfusion injury in mice. Lab Invest 89: 196-208, 2009.

7. Aydin SI, Seiden HS, Blaufox AD, Parnell VA, Choudhury T, Punnoose A and Schneider J: Acute kidney injury after surgery for congenital heart disease. Ann Thorac Surg 94: 1589-1595, 2012.

8. Garbaisz D, Turoczi Z, Aranyi P, Fulop A, Rosero O, Hermesz E, Ferencz A, Lotz G, Harsanyi L and Szijarto A: Attenuation of skeletal muscle and renal injury to the lower limb following ischemia-reperfusion using mPTP inhibitor NIM-811. PLoS One 9: e101067, 2014.

9. Sun Q, Meng QT, Jiang Y and Xia ZY: Ginsenoside Rb1 attenuates intestinal ischemia reperfusion induced renal injury by activating Nrf2/ARE pathway. Molecules 17: 7195-7205, 2012.

10. Nadkarni GN, Patel AA, Konstantinidis I, Mahajan A, Agarwal SK, Kamat S, Annapureddy N, Benjo A and Thakar CV: Dialysis requiring acute kidney injury in acute cerebrovascular accident hospitalizations. Stroke 46: 3226-3231, 2015.

11. Sheridan AM and Bonventre JV: Cell biology and molecular mechanisms of injury in ischemic acute renal failure. Curr Opin Nephrol Hypertens 9: 427-434, 2000.

12. Nicoud IB, Knox CD, Jones CM, Anderson CD, Pierce JM, Belous AE, Earl TM and Chari RS: 2-APB protects against liver ischemia-reperfusion injury by reducing cellular and mitochondrial calcium uptake. Am J Physiol Gastrointest Liver Physiol 293: G623-G630, 2007.

13. Lenoir O, Tharaux PL and Huber TB: Autophagy in kidney disease and aging: Lessons from rodent models. Kidney Int 90: 950-964, 2016.

14. Zhang YL, Zhang J, Cui LY and Yang S: Autophagy activation attenuates renal ischemia-reperfusion injury in rats. Exp Biol Med (Maywood) 240: 1590-1598, 2015. 
15. Decuypere JP, Ceulemans LJ, Agostinis P, Monbaliu D, Naesens M, Pirenne J and Jochmans I: Autophagy and the Kidney: Implications for Ischemia-Reperfusion Injury and Therapy. Am J Kidney Dis 66: 699-709, 2015.

16. Livak KJ and Schmittgen TD: Analysis of relative gene expression data using real-time quantitative PCR and the 2(-Delta Delta C(T)) method. Methods 25: 402-408, 2001.

17. Davenport A: AKI in a patient with cirrhosis and ascites. Clin J Am Soc Nephrol 7: 2041-2048, 2012.

18. Bagshaw SM, Cruz DN, Aspromonte N, Daliento L, Ronco F, Sheinfeld G, Anker SD, Anand I, Bellomo R, Berl T, et al: Epidemiology of cardio-renal syndromes: Workgroup statements from the 7th ADQI Consensus Conference. Nephrol Dial Transplant 25: 1406-1416, 2010.

19. West SC, Arulkumaran N, Ind PW and Pusey CD: Pulmonary-renal syndrome: A life threatening but treatable condition. Postgrad Med J 89: 274-283, 2013.

20. Ritz E: Intestinal-renal syndrome: Mirage or reality? Blood Purif 31: 70-76, 2011.

21. Lowe M: Structure and function of the Lowe syndrome protein OCRL1. Traffic 6: 711-719, 2005.

22. Tsagalis G, Akrivos T, Alevizaki M, Manios E, Theodorakis M, Laggouranis A and Vemmos KN: Long-term prognosis of acute kidney injury after first acute stroke. Clin J Am Soc Nephrol 4 616-622, 2009.

23. Khatri M, Himmelfarb J, Adams D, Becker K, Longstreth WT and Tirschwell DL: Acute kidney injury is associated with increased hospital mortality after stroke. J Stroke Cerebrovasc Dis 23: 25-30, 2014.

24. Ranganathan P, Jayakumar C, Mohamed R, Weintraub NL and Ramesh G: Semaphorin 3A inactivation suppresses ischemia-reperfusion-induced inflammation and acute kidney injury. Am J Physiol Renal Physiol 307: F183-F194, 2014.

25. Rabb H, Griffin MD, McKay DB, Swaminathan S, Pickkers P, Rosner MH, Kellum JA and Ronco C; Acute Dialysis Quality Initiative Consensus XIII Work Group: Inflammation in AKI: Current understanding, key questions and knowledge gaps. J Am Soc Nephrol 27: 371-379, 2016.

26. Nongnuch A, Panorchan K and Davenport A: Brain-kidney crosstalk. Crit Care 18: 225, 2014

27. Jin $X$, Zhang Y, Li X, Zhang J and Xu D: C-type natriuretic peptide ameliorates ischemia/reperfusion-induced acute kidney injury by inhibiting apoptosis and oxidative stress in rats. Life Sci 117: 40-45, 2014.

28. Elmore S: Apoptosis: A review of programmed cell death. Toxicol Pathol 35: 495-516, 2007.

29. Havasi A and Borkan SC: Apoptosis and acute kidney injury. Kidney Int 80: 29-40, 2011.
30. Ma P, Zhang S, Su X, Qiu G and Wu Z: Protective effects of icariin on cisplatin-induced acute renal injury in mice. Am J Transl Res 7: 2105-2114, 2015.

31. He L, Livingston MJ and Dong Z: Autophagy in acute kidney injury and repair. Nephron Clin Pract 127: 56-60, 2014.

32. Parzych KR and Klionsky DJ: An overview of autophagy: morphology, mechanism and regulation. Antioxid Redox Signal 20: 460-473, 2014.

33. Kaushal GP and Shah SV: Autophagy in acute kidney injury. Kidney Int 89: 779-791, 2016.

34. Jiang M, Liu K, Luo J and Dong Z: Autophagy is a renoprotective mechanism during in vitro hypoxia and in vivo ischemia-reperfusion injury. Am J Pathol 176: 1181-1192, 2010.

35. Chien CT, Shyue SK and Lai MK: Bcl-xL augmentation potentially reduces ischemia/reperfusion induced proximal and distal tubular apoptosis and autophagy. Transplantation 84: 1183-1190, 2007.

36. Hsiao HW, Tsai KL, Wang LF, Chen YH, Chiang PC, Chuang SM and Hsu C: The decline of autophagy contributes to proximal tubular dysfunction during sepsis. Shock 37: 289-296, 2012.

37. Kimura T, Takabatake Y, Takahashi A, Kaimori JY, Matsui I, Namba T, Kitamura H, Niimura F, Matsusaka T, Soga T, et al: Autophagy protects the proximal tubule from degeneration and acute ischemic injury. J Am Soc Nephrol 22: 902-913, 2011.

38. Sun H, Zou S, Candiotti KA, Peng Y, Zhang Q, Xiao W, Wen Y, $\mathrm{Wu} \mathrm{J}$ and Yang J: Octreotide attenuates acute kidney injury after hepatic ischemia and reperfusion by enhancing autophagy. Sci Rep 7: 42701, 2017

39. Zhang LX, Zhao HJ, Sun DL, Gao SL, Zhang HM and Ding XG: Niclosamide attenuates inflammatory cytokines via the autophagy pathway leading to improved outcomes in renal ischemia/reperfusion injury. Mol Med Rep 16: 1810-1816, 2017.

40. Cui J, Bai XY, Sun X, Cai G, Hong Q, Ding R and Chen X: Rapamycin protects against gentamicin-induced acute kidney injury via autophagy in mini-pig models. Sci Rep 5: 11256 , 2015.

41. Luo Z, Xu W, Ma S, Qiao H, Gao L, Zhang R, Yang B, Qiu Y, Chen J, Zhang M, et al: Moderate autophagy inhibits vascular smooth muscle cell senescence to stabilize progressed atherosclerotic plaque via the mTORC1/ULK1/ATG13 signal pathway. Oxid Med Cell Longev 2017: 3018190, 2017.

This work is licensed under a Creative Commons Attribution-NonCommercial-NoDerivatives 4.0 International (CC BY-NC-ND 4.0) License. 